\title{
Zinc and Cardio-Metabolic Health in Type 2 Diabetes Mellitus
}

\section{Samir Samman* and Meika Foster}

Discipline of Nutrition and Metabolism, School of Molecular Bioscience, University of Sydney, Australia

The essentiality of zinc in human nutrition was reported in the 1960s with the identification of young males who had short stature and exhibited hypogonadism. The root cause of the condition was traced back to the consumption of large quantities of dietary phytate, and the practice of geophagia, which was noted often among children in rural or pre-industrial societies. Both of these factors were thought to be responsible for inhibiting zinc absorption and ultimately lead to the diagnosis of zinc deficiency. We now recognize a plethora of symptoms associated with zinc deficiency that include high rates of infections, diverse forms of skin lesions, and impaired wound healing. These wide ranging symptoms point to the involvement of zinc in diverse cellular activities $[1,2]$. Other evidence implicates zinc in the etiologies of chronic diseases, particularly risk factors that are associated with cardio-vascular disease (CVD) and Type 2 diabetes mellitus (DM) $[3,4]$.

\section{Zinc Modulation of Glucose and Insulin Concentrations}

In a recent evaluation of randomized controlled clinical trials that were aimed at determining the effect of zinc supplementation on glycaemic control, we showed a small reduction in fasting glucose concentrations after zinc supplementation. In secondary analyses of participants with chronic metabolic diseases (Types 1 and $2 \mathrm{DM}$, metabolic syndrome, obesity), zinc supplementation resulted in a greater reduction in plasma glucose concentrations compared to the effect that was observed in healthy participants. These observations are supported further by other reports, mainly of cross-sectional surveys, which suggest that improved zinc status affects favorably the cardiometabolic profile in people with DM [5].

The potential mechanisms that are responsible for the observed effects on glycaemic control may be mediated through the association of zinc with insulin. Under physiological conditions, zinc is abundant throughout the pancreas, but is particularly concentrated in the secretary vesicles of the beta-cells where it forms an integral component of the insulin crystalline structure [6] serving to confer stability to the insulin granule [7]. Zinc transporter 8 (ZnT8) belongs to the CDF/ ZnT (SLC30) family of zinc transporters, and is abundantly expressed in the pancreas. ZnT8 co-localizes with insulin in pancreatic islets and appears to be involved in zinc accumulation and regulation of insulin secretion in beta-cells [8]. Recent evidence suggests that single nucleotide polymorphisms in ZnT8 are associated with impaired proinsulin conversion [9] and increased risk of developing type 2 DM $[10,11]$.

\section{Zinc and Plasma Lipoproteins}

Dyslipidemia is observed often in DM and poses an increased risk for CVD. The major change in the lipid profile of DM is an increase in plasma triglycerides and a reduction in High-Density Lipoproteins (HDL). HDL is responsible for cholesterol efflux from peripheral tissues and is considered to play an anti-atherogenic role [3]. Therefore, the decrease in HDL cholesterol, which is observed often in DM, is associated with increased risk of CVD [3].

In a meta-analysis of controlled clinical trials, we determined the effect of zinc supplementation on plasma lipoprotein cholesterol and triglyceride concentrations in humans [12]. No overall significant effects of zinc supplementation were observed for plasma cholesterol,
Low-Density Lipoprotein (LDL) cholesterol, HDL cholesterol or plasma triglyceride concentrations. Secondary analyses based on health status revealed that in subjects with DM or in those undergoing haemodialysis, zinc supplementation was associated with an increase in HDL cholesterol concentrations (Figure 1). When compared to baseline values, the mean change in plasma HDL in participants with type $2 \mathrm{DM}$, was $34 \%$, conferring a significant decrease in CVD risk.

The mechanisms may involve zinc's role in insulin action, which has been proposed as an independent predictor of plasma HDLcholesterol concentrations [13]. Moreover, as with glucose metabolism, many of the effects of insulin on lipid metabolism are mediated by the PI3K/Akt pathway and its regulation of FoxO-dependent gene transactivation. There are many roles ascribed to FoxO proteins in lipid metabolism that might conceivably be affected by changes to zinc homeostasis. These include the stimulation of lipoprotein lipase expression, the suppression of lipogenesis in the liver, and the transcriptional regulation of microsomal triglyceride transfer protein, which is involved in lipoprotein assembly [4].

The view that zinc may have a beneficial effect in DM and CVD is supported further by the observation that a higher plasma zinc concentration protects those with type $2 \mathrm{DM}$ from CVD complications [14]. In the meta-analysis [12], the increase in plasma zinc after zinc supplementation was significantly higher in those with type $2 \mathrm{DM}$ than in healthy subjects, despite these two groups having similar plasma zinc concentrations at baseline, suggesting that an underlying defect

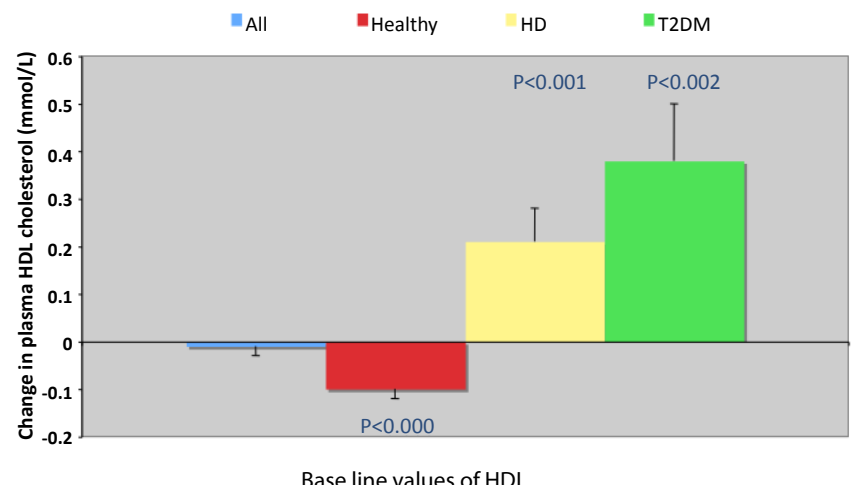

Figure 1: The effects of zinc supplementation on plasma HDL cholesterol concentrations in apparently healthy individuals, and those with Type 2 diabetes mellitus (T2DM) or undergoing renal dialysis (HD).

*Corresponding author: Samir Samman, Discipline of Nutrition and Metabolism, School of Molecular Bioscience, University of Sydney, Australia, E-mail: samir.samman@sydney.edu.au

Received November 12, 2012; Accepted November 14, 2012; Published November 21, 2012

Citation: Samman S, Foster M (2013) Zinc and Cardio-Metabolic Health in Type 2 Diabetes Mellitus. Vitam Trace Elem 2:e116. doi:10.4172/2167-0390.1000e116

Copyright: (c 2013 Samman S, et al. This is an open-access article distributed under the terms of the Creative Commons Attribution License, which permits unrestricted use, distribution, and reproduction in any medium, provided the original author and source are credited. 
in zinc homeostasis which may have a significant effect on lipoprotein metabolism.

\section{Zinc, Oxidative Stress and Antioxidant Function}

Reactive oxygen species (ROS) have important physiological roles in a wide range of signaling pathways, but their accumulation can place cells in a state of oxidative stress. Increased oxidative stress is related to key atherogenic events, including endothelial dysfunction, modulation of phosphorylation signaling; disturbances in redox homeostasis have been linked to chronic inflammation. These processes appear to be influenced by intracellular zinc concentrations [4].

Perturbed zinc homeostasis in DM leads to a state of zinc deficiency as evidenced in part by hyperzincuria that is consistently reported in the disease. Further loss of zinc, which is induced by oxidative stress, enhances the release of cellular zinc in insulin-responsive tissue [15]. One of the possible effects of low zinc availability is a reduction in superoxide dismutase (CuZnSOD) activity. It has been proposed that CuZnSOD protects insulin and beta-cells from oxidative damage [4]. Further, CuZnSOD, by preserving nitric oxide (NO), promotes glucose uptake by skeletal muscle and facilitates the binding of insulin to its receptor [4].

Despite being redox-inert, zinc exhibits a variety of indirect antioxidant effects. Zinc enhances the antioxidant capacity of the cell through direct competition with metals that are known to catalyze the Fenton reaction, such as copper and iron. In addition to being an integral component of SOD, zinc is able to induce the synthesis of metallothionein and glutathione, all of which protect against an accrual of reactive species in cellular systems. Further, zinc modulates the functions and interactions of numerous redox-responsive proteins at several levels of signaling cascades, and may itself act as a signaling ion [4].

\section{Zinc and Chronic Inflammation}

Intracellular zinc concentrations have been shown to influence the induction of inflammatory cytokines that regulate acute inflammation and which also are known to generate ROS [4]. Sustained expression of pro-inflammatory cytokines and impaired signaling in inflammation and oxidative stress contribute to the pathology of CVD and DM. Perturbations of zinc homeostasis are also associated with these disorders, suggesting an interrelationship between zinc, inflammation, and oxidative stress [16].

In human intervention studies that report the effects of zinc on plasma cytokines or their production in primary human blood cells, zinc supplementation decreases pro-inflammatory cytokine mRNA and protein synthesis in stimulated mononuclear cells $[4,16]$. Conversely, increased cytokine levels have been shown in stimulated mononuclear cells isolated from zinc-supplemented populations [16], suggesting a dose effect of zinc. A number of studies have investigated the relationship between zinc and C-reactive protein (CRP) concentrations in humans. In observational studies, there appears to be an inverse association between CRP and serum zinc concentrations [16]. Similarly, the effects of zinc on NF-kB activity appear to depend on the zinc status of the host. In humans, NF-kB activation was decreased in the peripheral blood mononuclear cells of elderly subjects with plasma zinc levels below the reference range. These effects were corrected with zinc supplementation.

\section{Zinc Biomarkers}

The concentration of zinc in serum (or plasma) is used commonly as a biomarker of zinc status. The concentration of zinc in serum represents less than $1 \%$ of the total metabolic pool of zinc; hence, its measurement provides limited information about the zinc status of individuals [17]. Several factors are known to affect the concentration of zinc in serum that include technical factors in the measurement as well as biological aspects such as: certain genotypes or mutations, age, gender, diurnal variations, underlying nutritional status, and interactions of zinc with drugs, including oral contraceptive agents, or nutrients. More recently identified factors are infection and inflammation that can be significant confounders in some population groups [18]. The absence of a clear biomarker presents a challenge when examining the relationship between zinc and chronic disease for the most part, trials have relied on supplementation studies, where it can be assumed that the participants' zinc status will increase during the intervention.

\section{Conclusion}

Lifestyle modification, dietary and pharmacological therapies remain a priority in the management of DM. However, impaired zinc homeostasis is prominent in chronic metabolic disorders including $\mathrm{DM}$ and CVD, and there are significant improvements in glucose and HDL-cholesterol concentrations following zinc supplementation. Furthermore, zinc may exert other protective effects by decreasing inflammation and oxidative stress. Collectively, these effects suggest that zinc status is an important consideration in the management of DM.

\section{References}

1. Shils ME, Shike M, Ross AC, Caballero B, Cousins RJ (2006) Modern Nutrition in Health and Disease. (10thedn), Lippincott Williams and Wilkins, Philadelphia: 271-285.

2. Samman S (2007) Zinc Nutr Diet 64: S131-134.

3. Foster M, Samman S. (2011) Zinc and atherosclerosis: clinical observations and potential mechanisms. In: Zinc in Human Health. 76: 347-372.

4. Foster M, Samman S (2010) Zinc and redox signaling: perturbations associated with cardiovascular disease and diabetes mellitus. Antiox Redox Signal 13: 1549-1573.

5. Capdor J, Foster M, Petocz P, Samman S (2012) Zinc and glycemic control: A meta-analysis of randomised placebo controlled supplementation trials in humans. J Trace Elem Med Biol 120-124.

6. Scott DA (1934) Crystalline insulin. Biochem J 28: 1592-1602

7. Vallee BL (1959) Biochemistry, physiology and pathology of zinc. Physiol Rev 39: 443-490.

8. Chimienti F, Devergnas S, Pattou F, Schuit F, Garcia-Cuenca R, et al. (2006) In vivo expression and functional characterization of the zinc transporter ZnT8 in glucose-induced insulin secretion. J Cell Sci 119: 4199-4206.

9. Kirchhoff K, Machicao F, Haupt A, Schäfer SA, Tschritter O, et al. (2008) Polymorphisms in the TCF7L2, CDKAL1 and SLC30A8 genes are associated with impaired proinsulin conversion. Diabetologia 51: 597-601.

10. Sladek R, Rocheleau G, Rung J, Dina C, Shen L, et al. (2007) A genome-wide association study identifies novel risk loci for type 2 diabetes. Nature 445: 881 885.

11. Kanoni S, Nettleton JA, Hivert MF, Ye Z, van Rooij FJ, et al. (2011) Total zinc intake may modify the glucose-raising effect of a zinc transporter (SLC30A8) variant: a 14-cohort meta-analysis. Diabetes 60: 2407-2416.

12. Foster M, Petocz P, Samman S (2010) Effects of zinc on plasma lipoprotein cholesterol concentrations in humans: a meta-analysis of randomised controlled trials. Atherosclerosis 210: 344-352.

13. Zavaroni I, Dall'Aglio E, Bruschi F, Bonora E, Pezzarossa A, et al. (1985 Evidence for an independent relationship between plasma insulin and concentration of high density lipoprotein cholesterol and triglyceride. Atherosclerosis 55: 259-266. 
Citation: Samman S, Foster M (2013) Zinc and Cardio-Metabolic Health in Type 2 Diabetes Mellitus. Vitam Trace Elem 2:e116. doi:10.4172/21670390.1000 e116

14. Soinio M, Marniemi J, Laakso M, Pyörälä K, Lehto S, et al. (2007) Serum zinc level and coronary heart disease events in patients with type 2 diabetes. Diabetes Care 30: 523-528.

15. Haase H, Maret W (2005) Fluctuations of cellular, available zinc modulate insulin signaling via inhibition of protein tyrosine phosphatases. J Trace Elem Med Biol 19: 37-42.

16. Foster M, Samman S (2012) Zinc and regulation of inflammatory cytokines: implications for cardiometabolic disease. Nutrients 4: 676-694.
17. Hess SY, Peerson JM, King JC, Brown KH (2007) Use of serum zinc concentration as an indicator of population zinc status. Food Nutr Bull 28: S403-S429

18. Raiten DJ, Namasté S, Brabin B, Combs G Jr, L'Abbe MR, et al. (2011) Executive summary--biomarkers of nutrition for development: building a consensus. Am J Clin Nutr 94: 633S-650S. 